\title{
BIBLIOGRAFIA PARCIAL
}

\author{
Christiane Stallaert \\ Universidades de Antuérpia e Leuven \\ Christiane.Stallaert@soc.kuleuven.be \\ christiane.stallaert@artesis.be
}

Evelyn Schuler Zea Universidade Federal de Santa Catarina evelynsz@gmail.com

A bibliografia aqui selecionada, introdutória e parcial, visa elencar alguns dos títulos nacionais e estrangeiros que versam, de forma direta ou indireta, sobre questões nas zonas de contato entre antropologia e tradução.

ASAD, Talal. (1986) "The Concept of Cultural Translation in British Social Anthropology", In: CLIFFORD, James \& George E. MARCUS (eds.) (1986) Writing Culture: The Poetics and Politics of Ethnography. Berkeley: University of California Press, 141-164.

BACHMANN-MEDICK, Doris (ed.). (2009) "Introduction. The Translational Turn”, Translation Studies, Vol.2, $\mathrm{N}^{\circ} 1,2-16$

BACHMANN-MEDICK, Doris. (2006) Cultural Turns. Neuorientierungen in den Kulturwissenschaften. Hamburg: Rowohlt. 
BACHMANN-MEDICK, Doris (ed.). (1997) Übersetzung als Repräsentation fremder Kulturen. Berlin: E. Schmidt.

BACHMANN-MEDICK, Doris (ed.). (1996) Kultur als Text. Die anthropologische Wende in der Literaturwissenschaft. Frankfurt/ Main: Fischer Taschenbuch Verlag.

BAKER, Mona (ed.). (2009) Critical Readings in Translation Studies. London \& New York: Routledge.

BASSNETT, Susan \& TRIVEDI, Harish. (1999) Postcolonial Translation. Theory and Practice. London \& New York: Routledge.

BASSNETT, Susan. (1998) "Translating Across Cultures", In: HUNSTON, Susan (ed.), Language at Work, Multilingual Matters, 72-85.

BEIDELMAN, T. O. (1971) The Translation of Culture: Essays on E. Evans-Pritchard. New York: Barnes \& Noble, Inc.

BENJAMIN, Abdala Junior (org.). (2004) Margens da cultura: mestiçagem, hibridismo \& outras misturas. São Paulo: Boitempo.

BENJAMIN, Walter. (2010) "A tarefa-renúncia do tradutor": Clássicos da Teoria da Tradução. Volume 1: Alemão-Português (org.: Werner Heidermann). Florianópolis, Universidade Federal de Santa Catarina. Tradução de Susana Kampff Lages.

BERMAN, Antoine. (1984) L'épreuve de l'étranger. Culture et traduction dans l'Allemagne romantique. Gallimard.

BERMANN, Sandra \& WOOD, Michael (eds.). (2005) Nation, Language, and the Ethics of Translation. Princeton/Oxford: Princeton University Press. 
BHABHA, Homi K. (2012) The Location of Culture. $2^{\text {nd }}$ revised edition. Taylor \& Francis.

BORNEMAN, John \& HAMMOUDI, Abdellah. (2009) Being There. The Fieldwork Encounter and the Making of Truth. Berkeley/ Los Angeles/London: University of California Press.

BRISSET, Annie. (1996) A Sociocritique of Translation. Toronto: University of Toronto.

BUDEN, Boris, NOWOTNY, Stefan, SIMON, Sherry, BERY, Ashok \& CRONIN, Michael. (2009) "Cultural translation: An introduction to the problem, and Responses", Translation Studies, 2:2, 196-219.

BUDICK, Sanford \& Wolfgang ISER (eds.). (1996) The Translatability of Cultures: Figurations of the Space Between, Stanford University Press.

BÜHRIG, Kristin, HOUSE Juliane \& TEN THIJE, Jan D. (eds.). (2009) Translational Action and Intercultural Communication. Manchester: St Jerome Publishing.

BUZELIN, Hélène. (2004) "La traductologie, l'ethnographie et la production des connaissances”, Meta, XLIX, 4, 729-746.

BUZELIN, Hélène. (2005) "Unexpected allies: how Latour's network theory could complement bourdieusian analyses in translation studies". The Translator. v. 11 (2): 193-218.

CABRAL, João de Pina. (2003) "Semelhança e Verossimilhança: horizontes na narrativa etnográfica". Mana, vol 9 (1): 109-122.

CALLON, Michel. (1980) "Struggles and Negotiations to Define What Is Problematic and What Is Not: the Socio-Logic of 
Translation". In: K.D. KNORR \& R. KROHN \& R.D. WHITLEY (eds.). The Social Process of Scientific Investigation. Sociology of the Sciences, IV, Dordrecht/Boston: D. Reidel, 197-220.

CALLON, Michel. (1986) "Some elements of a sociology of translation: domestication of the scallops and the fishermen of St Brieuc Bay", first published in J. LAW, Power, action and belief: a new sociology of knowledge? London: Routledge, 196-223.

CARNEIRO DA CUNHA, Manuela. (1998) "Pontos de vista sobre a floresta amazônica: xamanismo e tradução”. Mana 4 (1): 7-23.

CHURCHILL JR., Christian J. (2005) "Ethnography as Translation”, Qualitative Sociology 28 (1): 3-24.

CLIFFORD, James \& George E. MARCUS (eds.). (1986) Writing Culture: The Poetics and Politics of Ethnography. Berkeley: University of California Press.

CLIFFORD, James. (1999) Routes: travel and translation in the late twentieth century. Cambridge, Mass.

CRONIN, Michael. (1996) Translating Ireland: Translation, Languages and Cultures. Cork: Cork University Press.

DE SOUSA SANTOS, Boaventura. (2006) The rise of the global left. The World Social Forum and beyond. London/New York: Zed Books.

DERRIDA, J. \& LÉVESQUE, C. (1982) L'oreille de l'autre: otobiographies, transferts, traductions, Montréal, VLB Editeur.

DERRIDA, Jacques. (1996) Le monolinguisme de l'autre ou La prothèse d'origine, Paris: Galilée. 
DERRIDA, Jacques. (2003) Torres de Babel. Belo Horizonte, UFMG. Tradução de Junia Barreto.

DINGWANEY, Anuradha \& Carol MAIER (eds.). (1995) Between Languages and Cultures. Translation and Cross-Cultural Texts. University of Pittsburgh Press.

FAULHABER, Priscila. (2008) "Etnografia na Amazônia e Tradução Cultural. Comparando Constant Tastevin e Curt Nimuendaju". Boletim do Museu Paraense Emílio Goeldi. Ciências Humanas, v. 3: 15-29.

FREIRE, José Ribamar Bessa. (2009) "Tradução e Interculturalidade: o passarinho, a gaiola e o cesto". Alea. Estudos Neolatinos, v. 11: 321-340.

GEERTZ, Clifford. (2003) “ 'Descoberto na Tradução': A História Social da Imaginação Moral" In: O Saber Local.Novos ensaios em antropologia interpretativa. Petrópolis: Vozes.

GEERTZ, Clifford. [1973] 2000. The Interpretation of Cultures. New York: Basic Books.

GEERTZ, Clifford; CLIFFORD, James et al. (1996) El surgimiento de la antropología posmoderna. Compilación de Carlos Reynoso. Barcelona: Gedisa, (Tercera Edición).

GLUCK, Carol \& Anna LOWENHAUPT TSING (eds.). Words in Motion. Toward a Global Lexicon. Durham/London: Duke University Press.

HALL, Kathleen D. (2002) Lives in Translation. Sikh Youth as British Citizens, University of Pennsylvania Press. 
HATIM, Basil. (1997) Communication across cultures: translation theory and contrastive text linguistics. University of Exeter Press.

HEILBRON, Johan. (1999) "Towards a Sociology of Translation: Book Translations as a Cultural World-System”, European Journal of Social Theory, 2 (4), 429-444.

HERMANS, Theo (ed.). (1985) The Manipulation of Literature. Studies in Literary Translation. London \& Sydney: Croom Helm.

HERMANS, Theo. (2002) Crosscultural Transgressions. Research Models in Translation Studies. St. Jerome Publishing.

HERMANS, Theo. (2006) Translating Others (2 Volumes). St. Jerome Publishing.

HUANG, Yunte. (2002) Transpacific Displacement: Ethnography, Translation, and Intertextual Travel in Twentieth-Century American Literature. Berkeley: University of California Press.

INGHILLERI, Moira (ed.). (2005) Bourdieu and the Sociology of Translation and Interpreting. The Translator. Studies in Intercultural Communication, Special Issue, Volume 11, Number 2.

JORDAN, Shirley Ann. (2002) "Ethnographic Encounters: The Processes of Cultural Translation", Language and Intercultural Communication, Volume 2, Issue 2, 96-110.

KATAN, David. (2004) Translating cultures. An Introduction for Translators, Interpreters and Mediators. Manchester: St. Jerome Publishing.

LATOUR, Bruno. (1989) “A. Traduire les intérêts des autres”. In: La science en action. Paris: La découverte. 
LAW, John \& John, HASSARD (eds.). (1999) Actor Network Theory and After. Oxford: Blackwell Publishing/The Sociological Review, (reprinted 2004, 2005).

LAW, John. (2003) Traduction/Trahison: Notes on ANT. Centre for Science Studies, Lancaster University, http://www.lancs. ac.uk/fass/sociology/papers/law-traduction-trahison.pdf $>$.

LEFEVERE, André (ed.). (1992) Translation/History/Culture. A Sourcebook. London and New York: Routledge.

LIENHARDT, Godfrey. (1963) "Modes of thought" In: The institutions of Primitive Society. A series of Broadcast Talks (ed: E. E. Evans-Pritchard). Oxford: Basil Blackwell.

MAHADEVAN, Jasmin. (2012) "Translating nodes of power through reflexive ethnographic writing", Journal of Organizational Ethnography, Vol. 1 Iss: 1, $119-131$.

MARANHÃO, Tullio \& Bernhard STRECK. (2003) Translation and Ethnography. The Anthropological Challenge of Intercultural Understanding. Tucson: University of Arizona Press.

MARCUS, George E. (1998) Ethnography through Thick and Thin. Princeton University Press.

MUNDAY, Jeremy (ed.). (2008) Translation as Intervention. Continuum Studies in Translation.

NEEDHAM, Rodney. (1972) Belief, Language and Experience. Oxford, Basil Backwell.

NEUMANN, Birgit \& NÜNNING Ansgar (eds). (2012) Travelling Concepts for the Study of Culture. Berlin, New York: de Gruyter. 
NIRANJANA, Tejaswini. (1992) History, Post- Struturalism and the Colonial Context. Siting Translation. Berkeley, University of California Press.

O'HAGAN, Minako \& David ASHWORTH. (2002) Translationmediated Communication in a Digital World. Facing the Challenges of Globalization and Localization. Multilingual Matters Ltd.

ORTEGA, Julio. (2006) Transatlantic translations : dialogues in Latin American literature, London: Reaktion Books.

OVERING , Joana. (1987) "Translation as a Creative Process: The Power of the Name". In: L. HOLY (ed.), Comparative Anthropology. Oxford: Basil Blackwell.

PALSSON, Gisli. (1994) Beyond Boundaries: Understanding, Translation and Anthropological Discourse. Explorations in Anthropology. Oxford: Berg.

POMPA, Cristina. (2003) Religião como tradução: missionários, Tupi e Tapuia no Brasil colonial. Bauru, SP: EDUSC/ANPOCS.

POVINELLI, Elizabeth A. (2001) "Radical Worlds: The Anthropology of Incommensurability, an Inconceivability". Anu. Rev. Anthrop. 30: 319-34.

PRATT, Mary Louise. (1999) Os olhos do império: relatos de viagem e transculturação. Bauru: EDUSC.

PRATT, Mary Louise, WAGNER, Birgit, CARBONELL I CORTÉS, Ovidi, CHESTERMAN, Andrew and TYMOCZKO, Maria. (2010) "Translation Studies Forum: Cultural translation", Translation Studies, 3: 1, 94-110 < http://dx.doi. org/10.1080/14781700903338706> 
PRICE, Joshua. (2007) "Lenguas híbridas, traducción y desafios poscoloniales", ÍKALA, revista de lenguaje y cultura, Vol. 12, N. ${ }^{\mathrm{o}} 18$.

PYM, Anthony. (2000) Negotiating the Frontier. Translators and Intercultures in Hispanic History. St. Jerome Publishing.

RICOEUR, Paul. (2006) On Translation. London and New York: Routledge.

ROBINSON, Douglas. (1997) Translation and Empire. Postcolonial Theories Explained. Manchester: St. Jerome Publishing.

RUBEL, Paula G. \& Abraham ROSMAN. (2003) Translating Cultures: Perspectives on Translation and Anthropology. Oxford, Berg.

SAVILLE-TROIKE, Muriel. (2003) The ethnography of communication: an introduction. 3rd ed. - Oxford: Blackwell.

SCHLEIERMACHER, Friedrich D.E. (2010) "Sobre os diferentes Métodos de Tradução". In: Clássicos da Teoria da Tradução. Volume 1: Alemão-Português (org.: Werner Heidermann). Florianópolis, Universidade Federal de Santa Catarina. Tradução de Celso R. Braida.

SCHULER ZEA, Evelyn. (2008) "Genitivo da Tradução", Boletim do Museu Paraense Emílio Goeldi. Ciências Humanas, Belém, v. 3(1): 65-77.

SENFT, Gunter, Jan-Ola ÖSTMAN \& Jef VERSCHUEREN (eds.). (2009) Culture and Language Use. Handbook of Pragmatics Highlights, Volume 2. John Benjamins Publishing. 
SILVA-SANTISTEBAN, Ricardo. (2010) "Garcilaso de la Vega Traductor", Mutatis Mutandis. Vol. 3, No. 2, 235-248.

SNELL-HORNBY, Mary. (2006) The Turns of Translation Studies. New paradigms or shifting viewpoints? Amsterdam/Philadelphia: John Benjamins Publishing Company.

SNELL-HORNBY, Mary; Zuzana JETTMAROVÁ; Zuzana Jettmarova \& Klaus KAINDL (eds.). (1997) Translation as Intercultural Communication: Selected Papers from the Est Congress, Prague 1995. John Benjamins Publishing.

STALLAERT, Christiane. (2006) Ni una gota de sangre impura. La España inquisitorial y la Alemania nazi cara a cara. Barcelona: Galaxia Gutenberg.

STALLAERT, Christiane. (2008) "Over de (on)vertaalbaarheid van het nazisme. De stem van de daders", Filter. Tijdschrift over Vertalen, 15 (4), 13-25.

STALLAERT, Christiane. (2009) "La comunicación intercultural y el holocausto: traducir o silenciar la voz del perpetrador", in: ALVAREZ SILVA, María Rosa; ALVAREZ MORENO, Celia; RUIZ MIYARES, Leonel. Actas del XI Simposio Internacional de Comunicación Social. Santiago de Cuba, 19-23 de enero de 2009. Centro de Lingüística Aplicada (Universidad de Oriente). Ministerio de Ciencia, Tecnología y Medio Ambiente. Santiago de Cuba, 368-372.

STALLAERT, Christiane. (2010) "Do antissemitismo emocional ao antissemitismo funcional na Espanha inquisitorial e na Alemanha nazista", in: TUCCI CARNEIRO, Maria Luiza \& Federico CROCI (eds.), Tempos de Fascismos. Ideologia - Intolerancia - Imaginário. São Paulo: Editora da Universidade de São Paulo, Imprensa Oficial, Arquivo Público do Estado de São Paulo, 37-71. 
STALLAERT, Christiane. (2010) "1492/1942, paisatges de memòria en l'Europa transmoderna", in: L'Espill, Segona Època, Núm. 36, Hivern 2010, 45-53.

STALLAERT, Christiane. (2011) "Castas, razas, etnias. Circulación intercontinental de conceptos y personas", in: VANDEN BERGHE, Kristien (ed.), El retorno de los galeones. Literatura, arte, cultural popular, historia. Frankfurt/Main: Peter Lang, 77-94.

STALLAERT, Christiane. (2012) 'Conversión religiosa y traducción en las culturas ibéricas. Dos aliados en la creación de identidades contra-hegemónicas', in: HENAO RESTREPO, Darío (comp.), X Jornadas Andinas de Literatura Latinoamericana. Fronteras e Interculturalidad. Actas de las, 30/7-3/8/2012. Cali: Universidad del Valle, 536-548.

STALLAERT, Christiane. (2012, forthcoming) "Nuevos patrones de identidad y memoria en la Europa transmoderna", Ilha Revista de Antropologia, Universidade Federal de Santa Catarina - UFSC, Florianópolis, SC, Brasil.

STALLAERT, Christiane. (2012, forthcoming) "Translation and Conversion as Interconnected 'Modes'. A Multidisciplinary Approach to the Study of Ethnicity and Nationalism in Iberian cultures". In: RESINA, Joan Ramon, Iberian Modalities. Liverpool University Press, 135-152.

STALLAERT, Christiane. (2013, forthcoming) "Discursos, políticas y prácticas de convivencia en la Europa del siglo XXI. Una traducción antropológica”, Gazeta de Antropología, Universidad de Granada, 30.

STALLAERT, Christiane. (2013, forthcoming) "Hybridization, Transculturation, and Translation. Europe through the Lens of 
Latin America", in: SABATÉ, Flocel (ed.), Hybrid Identities. An interdisciplinary vision of social mobility. Turnhout: Brepols Publishers.

STALLAERT, Christiane. (2013, forthcoming) "Traducción y Transmodernidad, herramientas de análisis de procesos de transculturación en un mundo globalizado", in: Actas del XIII Simposio de Comunicación Social, Santiago de Cuba: Universidad de Oriente.

STALLAERT, Christiane. (forthcoming) 'A crise do multiculturalismo na Europa. Reflexões em torno do conceito de 'convivência". In: TUCCI CARNEIRO, Maria Luiza (ed.), Intolerância em Tempos de Liberdade e Democracia. Actas do I Forum de Combate ao Racismo, à Xenofobia e à Discriminação (2526 de Maio de 2011). São Paulo: LEER / FISESP.STURGE, Kate (1997) "Translation Strategies in Ethnography", The Translator. Vol. 3, No.1, 21-38.

STURGE, Kate. (2007) Representing Others. Translation, Ethnography and the Museum (Translation Theories Explored 11). Manchester: St. Jerome Publishing.

TAMBIAH, Stanley Jeyarara. (1995) "Rationality, relativism the translation and commensurability of cultures", in: Magic, Science and the scope of rationality. Harvard, Cambridge University Press.

TEDLOCK, Dennis \& Bruce MANNHEIM (eds.). (1995) The dialogic Emergence of Culture. Urbana and Chicago: University of Illinois Press.

TRANSVERSAL, Borders, Nations, Translations, http://eipcp. net/transversal/0608 
TYMOCZKO, Maria. (2007) Enlarging Translation, Empowering Translators. Manchester: St. Jerome Publishing.

VALERO-GARCÉS, Carmen. (1995) "Modes of Translating Culture: Ethnography and Translation", Meta, vol. 40, n 4, 556563.

VENUTI, Lawrence. (1992) Rethinking Translation: Discourse, Subjectivity, Ideology. Taylor \& Francis.

VENUTI, Lawrence. (1995) The translator's invisibility: a history of translation. London: Routledge.

VENUTI, Lawrence. (1998) The scandals of translation: towards an ethic of difference. London: Routledge.

VENUTI, Lawrence (ed.). (2000) The Translation Studies Reader. London and New York: Routledge.

VIVEIROS DE CASTRO, Eduardo. (2004) "Perspectival Anthropology and the Method of Controlled Equivocation". Tipiti 2: 3-22.

WERNER, Oswald \& CAMPBELL, Donald T. (1970) "Translating, Working Through Interpreters, and the Problem of Decentering". In: NARON, Raoul and COHEN, Ronald (eds): A Handbook of Method in Cultural Anthropology. New York: Columbia University Press.

WOLF, Michaela \& FUKARI, Alexandra. (2007) Constructing $a$ Sociology of Translation. Amsterdam/Philadelphia: John Benjamins Publishing Company. 
WOLF, Michaela. (2008) "Translation - Transculturation. Measuring the perspectives of transcultural political action", Translated by Kate Sturge, Transversal, Borders, Nations, Translations, http://eipcp.net/transversal/0608/wolf/en.

WOLF, Michaela. \& FUKARI, Alexandra (eds.). (2007) Constructing a Sociology of Translation. Amsterdam: John Benjamins Publishing Company. 